\title{
Assessing soil water content variability through active heat distributed fiber optic temperature sensing
}

\author{
Sergio Zubelzu ${ }^{\mathrm{a}, *}$, Leonor Rodriguez-Sinobas ${ }^{\mathrm{a}}$, Antonio Saa-Requejo ${ }^{\mathrm{b}, \mathrm{c}}$, Javier Benitez ${ }^{\mathrm{a}}$, \\ Ana M. Tarquis ${ }^{c, d}$ \\ a Grupo de Investigación "Hidráulica del Riego", E.T.S.I. Agronómica, Alimentaria y Biosistemas, Universidad Politécnica de Madrid, Ciudad Universitaria s/n, 28040, \\ Madrid, Spain \\ ${ }^{\mathrm{b}}$ Departamento de Producción Agraria, E.T.S.I. Agronómica, Alimentaria y Biosistemas, Universidad Politécnica de Madrid, Ciudad Universitaria s/n, 28040, Madrid, \\ Spain \\ c CEIGRAM, Universidad Politécnica de Madrid, C/ Senda del Rey 13, Ciudad Universitaria Madrid, 28040, Spain \\ d Grupo de Sistemas Complejos, Universidad Politécnica de Madrid, Ciudad Universitaria s/n, 28040, Madrid, Spain
}

\section{A R T I C L E I N F O}

\section{Keywords:}

Multiscaling analysis

Generalized structure function

Soil moisture variability

Fiber optic cable

Statistical moments

Fluctuations

\begin{abstract}
A B S T R A C T
Soil spatial variability is a key point for the sustainable water management in agriculture. Fractal techniques provide proper tools to analyze soil spatial variability searching for statistical self-similarity patterns among different scales. Although they have been extensively applied to study the soil properties variability, its applicability for the soil water content (SWC) distribution is complicated because requires many data difficult to obtain with the typical point soil water sensors. Recently, a fiber optic distributed temperature sensor has been used to measure soil thermal properties which relate to SWC. These sensors provide large amount of data with high spatial and temporal resolution, thus filling the gap of point soil water sensors. In the present work, soil temperature was measured with a Distributed Temperature Sensing (DTS) and SWC was estimated by different fitting functions which have been studied with focus on spatial variability.

Temperature was measured in a $133 \mathrm{~m}$ fiber optic cable laid in a sandy soil field plot. A The Active Heat Fiber Optic (AHFO) method was used, with $12 \mathrm{~cm}$ sampling resolution, and heat pulses $(19,4 \mathrm{~W} / \mathrm{m}$ during 2 min) were applied. The temperature data were correlated to SWC, considering the integration of temperature during the heat pulse $\mathrm{T}_{\text {cum }}$, and then the datasets Tcum-SWC were fitted to the best fit statistical function (exponential, potential and polynomial). The results showed that the $\mathrm{T}_{\text {cum }}$ distribution presented a non-Gaussian pattern. Additionally, highly anti-persistent patterns have been detected for the larger spatial scaling lags. The function`s performance was different thus, the exponential function reproduced better the absolute moments of the temperature profile but it failed reproducing the non-Gaussian behavior.
\end{abstract}

\section{Introduction}

Agriculture is the largest water user with approximately $70 \%$ of the global consumption (Fischer et al., 2007). Soil water content (SWC) represents a small percentage $(\approx 0.05 \%)$ of the global freshwater resources (Dingman, 2002) but it is a key factor that conditions the agricultural yields. Good agricultural performance, especially in irrigated areas, is limited by the SWC and its availability for the plants. SWC measurements for irrigation management are commonly obtained from point sensors. Although some works have dealt with the proper sensor placement (Dabach et al., 2015), they have not consider soil water spatial variability whose impact on SWC distribution has been widely assessed (Burrough et al., 1994).
Soil spatial variability has been studied using different theoretical frameworks. Thus, geostatistics have been used for quantifying the spatial pattern of soil properties, and Kriging techniques have been proved sufficiently robust for estimating values at non sampled locations in most of the cases (Nielsen and Wendroth, 2003; Webster and Oliver, 2001).

Multifractal analysis (MFA) was initiated with the seminal work by Mandelbrot (1982) and can provide insight into spatial variability of crop or soil parameters (Vereecken et al., 2007). This technique has been used to characterize the scaling property of a variable measured along a transect as a mass distribution of a statistical measure on a spatial domain of the studied field (Zeleke and Si, 2004; Lopez de Herrera et al., 2016 among others). Based on rescaled range analysis

\footnotetext{
* Corresponding author.

E-mail address: sergio.zubelzu@upm.es (S. Zubelzu).
} 


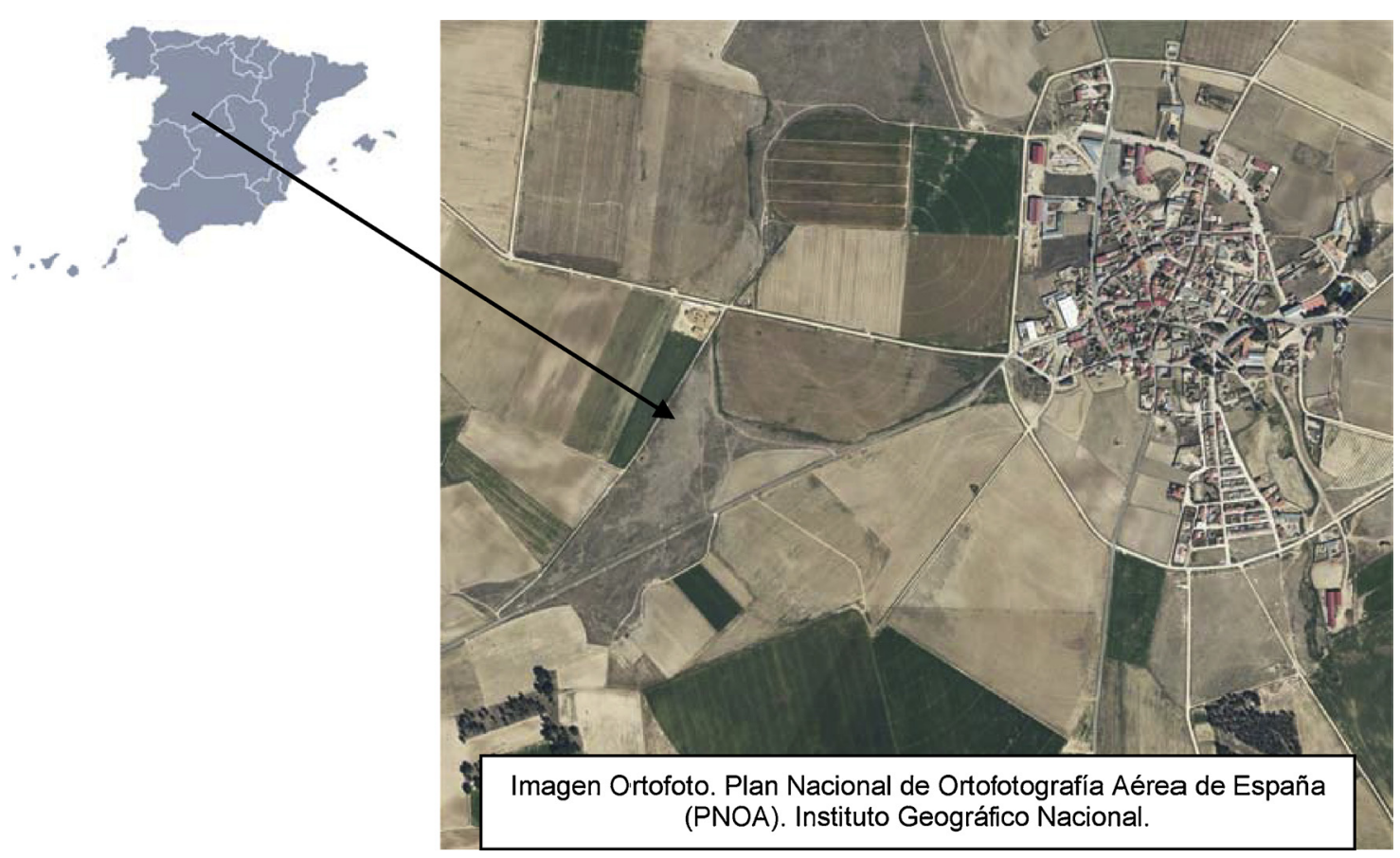

Fig. 1. Location of the experimental plot (Image (C) 2018 Google, Data from map (C) 2018 Google, Instituto Geográfico Nacional).

(Hurst, 1951), several MFA can be used to study the fluctuation in signals (Davis et al., 1994; Schmitt et al., 1995; Taqqu et al., 1995).

Some authors have applied these techniques to soil science to look for statistical self-similar series (Pozdnyakova et al., 2005; Kravchenko et al., 2002; Garcia Moreno et al., 2010; Morató et al., 2017). Likewise, other works (Tarquis et al., 2008; Morató et al., 2017) have studied wider scaling behaviors which cannot be captured in a consistent way by the MFA. These analyses include linear relations between log structure functions, and frequency distributions of the variables' increments tending to be symmetric with increasing kurtosis as the lags between pairs of values decreases (Guadagnini et al., 2015; Molinari et al., 2015).

Despite of the many studies focused on the MFA of soil properties, only few have centered on applying these techniques to study SWC spatial variability. Their application is limited by the use of discrete measurements by point sensors, and consequently the high cost of collecting enough data.

However in this century, the development of approaches to estimate SWC by distributed temperature techniques (DTS) can help to fill this gap. They estimate SWC from a distributed temperature measurement along a fiber optic (FO) cable. Its resolution depends on the cable's properties and the DTS unit, limiting the cable's length that can be deployed up to several kilometers (Selker et al., 2006). Soil temperature and therefore SWC, can be obtained every second and every $12 \mathrm{~cm}$.

Among these approaches, both passive and active methods (AHFO) have been proposed to estimate SWC from the temperature measurement of the FO cable. While passive techniques only require the simple temperature measurement, AHFO requires heating the FO cable to study the heat transfer pattern from the cable to the surrounding soil to be correlated with the SWC. The procedure determines the best fitting curve between the heat transfer indicator and the SWC and then, each value of the heat transfer indicator measured in the field will be easily transformed into SWC.

Several indicators for heat pattern transfer are available. For example, Sayde et al (2010) or Striegel and Loheide (2012) proposed the $\mathrm{T}_{\text {cum }}$ or $\Delta \mathrm{T}_{8}$, respectively. In addition, some fitting curves have also been defined to correlate them with SWC (Serna et al., 2017; Gil Rodríguez et al., 2013; Benitez-Buelga et al., 2016). However, there are some uncertainties that must be taken into account not only when calculating the fitting curve but also in the SWC estimation. For example, some authors determine the fitting curve trough measurements in soil disturbed samples in the laboratory that later will be used to estimate the SWC in field experiments. Alternatively, other authors determine it in the field without disturbing the soil structure. The fitting functions obtained in the lab usually cover the whole range of possible SWC, from the residual to the saturated levels. Conversely, their determination in the field often only covers a narrower SWC range.

Otherwise, the authors do not reach an agreement on the functional form of the estimation fitting curve. Potential, polynomial end exponential have majorly been proposed. The differences among them are evident and their selection will affect the accuracy of SWC estimation.

So that, there is a scope for further analysis related to the SWC spatial variability and to the procedures to estimate the SWC from the DTS measurements.

Within this framework, this paper focuses on the study of SWC spatial variation, using DTS, and on the SWC estimation from the DTS as well. The main objectives are (1) to study the spatial variability of the SWC using the data collected from the DTS and (2) to discuss the SWC estimation procedures from the indicators obtained with the DTS technique.

\section{Materials and methods}

\subsection{Experimental sites}

The study site was a field plot of $300 \mathrm{~m}^{2}$ of sandy soil located in the Irrigation District "Río Adaja", Nava de Arévalo (Ávila, Spain), (Fig. 1).

The experimental installation comprised a buried FO cable, a DTS and an electrical installation to heat the FO cable (Fig. 2).

The FO cable, $300 \mathrm{~m}$ long, was buried at 0.2 and $0.4 \mathrm{~m}$ depths laid in 6 parallel with symmetric spacing between successive pairs of parallel cables (Fig. 3).

The cable's deployment was conducted during the first week of August 2010. FO cable was buried helped by a custom plow with two blades held at 45-degrees from vertical that simultaneously buried cables at $0.2 \mathrm{~m}$ and $0.4 \mathrm{~m}$ depths. These depths were chosen to monitor SWC within the root's zone for most crops at the location. The cable's diameter was $3.8 \cdot 10^{-3} \mathrm{~m}$ comprising 3 layers of sheathing, a stainless 


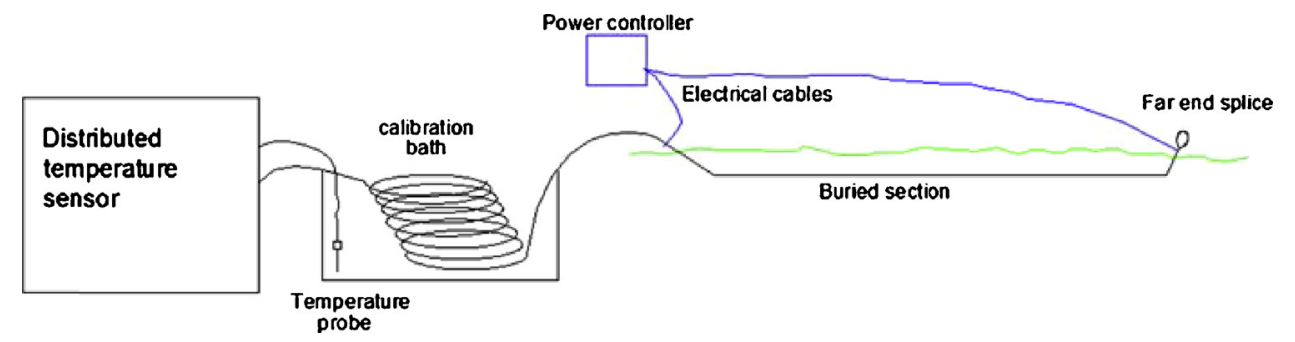

Fig. 2. Sketch of the fiber optic cable installation.

(Adapted from Benitez-Buelga et al., 2016).

steel jacket and four fiber optic lines (Brugg, Switzerland).

The site was maintained for two years as bare soil in order to ensure the perfect contact between the cable and the soil and to let the soil settle.

The DTS unit was a Silixa Ultima SR (Silixa Ltd, UK) with sampling resolution of $0.125 \mathrm{~m}$ and temporal resolution of $1 \mathrm{~s}$. A double ended set up was achieved by connecting the beginning of both cables to the DTS unit and by splicing the far ends of the FO lines (Fig. 2). Approximately $20 \mathrm{~m}$ of FO cable at the beginning of the installation were reserved for DTS calibration (Fig. 2).

For the Active Heated Fiber Optic (AHFO) experiment, two electrical cables were connected to the metallic jacket of the fiber optic cable (Fig. 3). A voltage potential difference was applied between connections in order to supply a constant power along the heated section of the FO cable. The intensity of the heat pulses was monitored by electronically controlled switches (Single phase micro fusion power controller, Control Concepts Inc.).

In order to obtain enough SWC content data to deduce the estimation fitting curves (see Section 2.2), SWC was monitored at two sampling areas with two ECH2O 5 TM dielectric probes (Decagon, Pullman, WA, USA).

\subsection{Active fiber optic measurements}

The DTS employs light's pulses in the infrared spectrum propagating in the FO cable. It focusses on the backscattered light called Raman spectra which consist of two signals of wavelengths: Stokes and Anti- Stokes. The second, unlike the stokes, are sensitive to the surrounding temperature, which is the temperature measurement principle. We use the $\mathrm{T}_{\text {cum }}$ (Eq. (1)) proposed by Sayde et al. (2010) as the heat transfer indicator from which estimate SWC.

$T_{\text {cum }}=\int_{t_{0}}^{t_{1}} \Delta T d t$

In which $\mathrm{T}$ is the temperature and t time. Fig. 4 shows the graphical representation of the $T_{\text {cum }}$ and the evolution of the temperature during the experimental runs.

In the literature, different fitting curves have been used to relate $\mathrm{T}_{\text {cum }}$ and SWC with different experimental setups: Benitez-Buelga et al. (2016) fitted a polynomial curve relating SWC with $\mathrm{T}_{\text {cum }}$ using observed data coming from the site of the study. The resulting curve is presented in Eq. (2).

$T_{\text {cum }}=\frac{1}{a+b S W C+c / S W C}$

Where $\mathrm{a}, \mathrm{b}$ and $\mathrm{c}$ are numerical parameters. The $\mathrm{R}^{2}$ of the fitting curve was 0.99 . From this relation we expressed SWC as follows to use in this study (Eq. (3)).

$S W C=\frac{\left(\frac{1}{T_{\text {cum }}}-a\right)+\sqrt{\left(\frac{1}{T_{\text {cum }}}-a\right)^{2}-4 b c}}{2 b}$

This relation is named as the polynomial model estimation of SWC (SWCpoly). The values of the parameters are $\mathrm{a}=8410^{-5}, \mathrm{~b}=2610^{-5}$ and $\mathrm{c}=1.610^{-5}$

Serna et al. (2017) used a potential function to relate $\mathrm{T}_{\text {cum }}$ and SWC based on the experimental data obtained in a laboratory experiment using sandy soil (Eq. (4)).

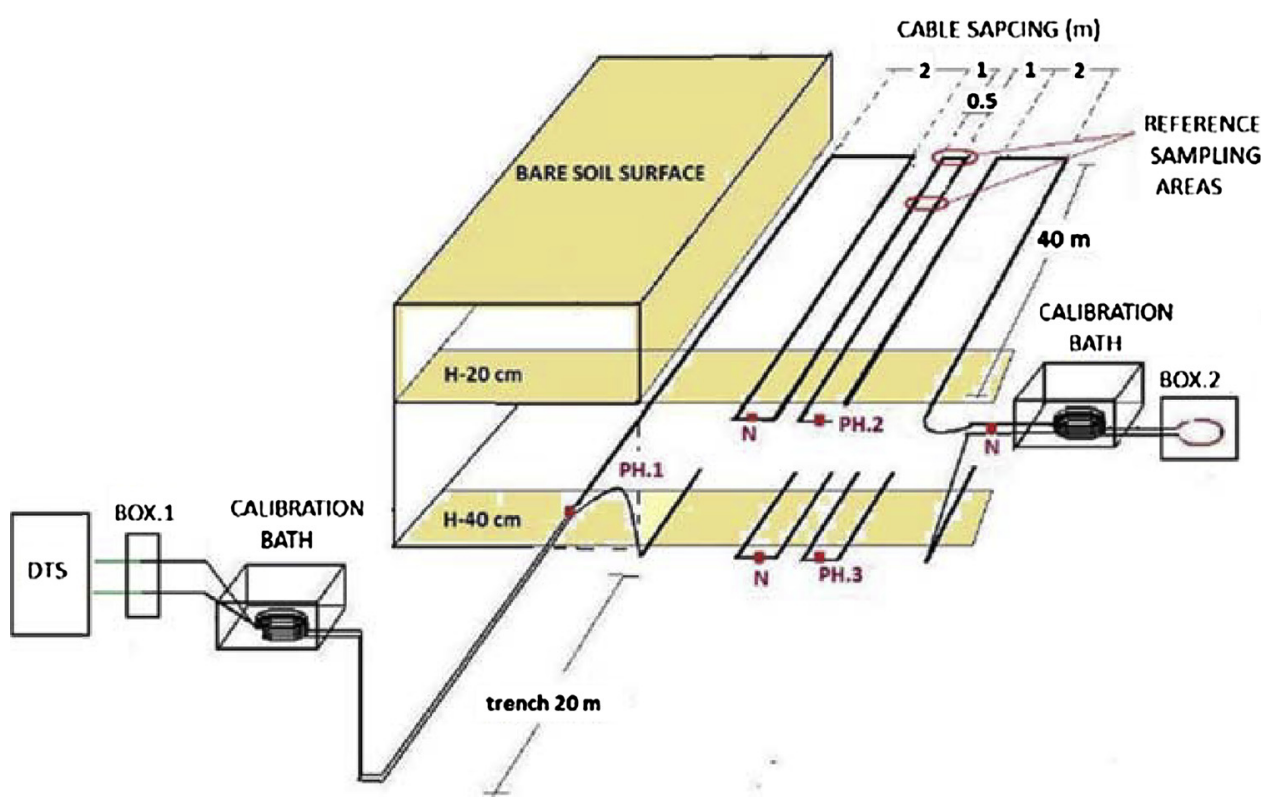

Fig. 3. Scheme of the experimental set up. 


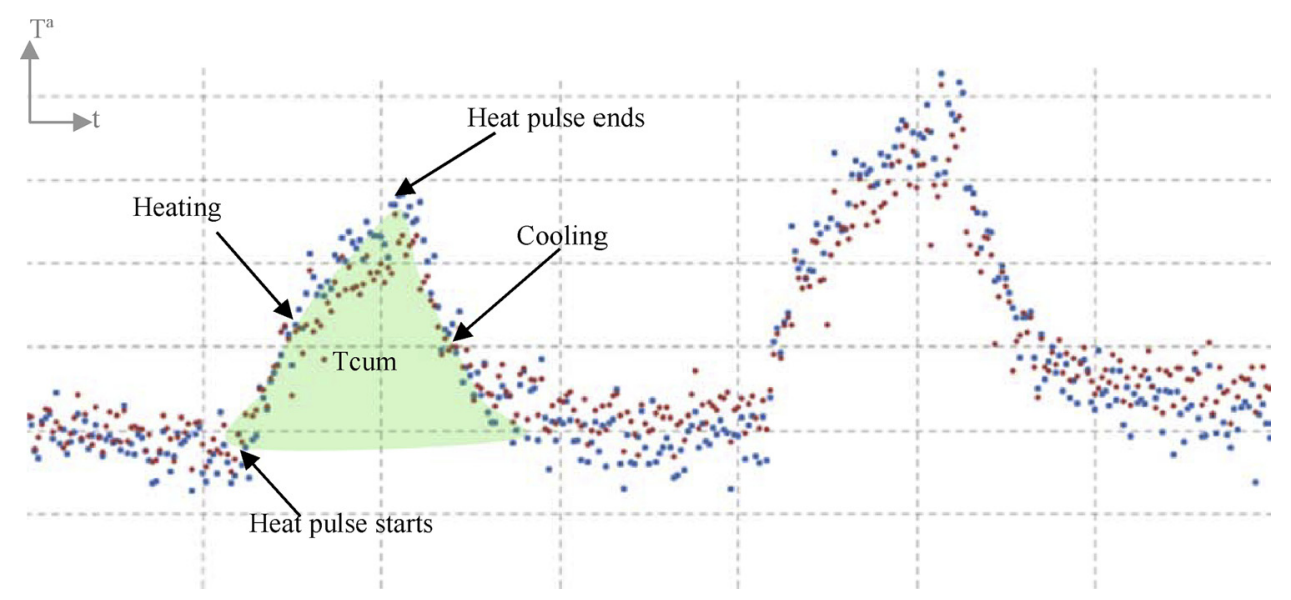

Fig. 4. Graphical meaning of Tcum and temperature evolution during the experiments.

$S W C=k T_{c u m}^{-d}$

In which $k$ and $d$ are numerical parameters which amounted to $\mathrm{k}=810^{34}, \mathrm{~d}=11.72$ and an $\mathrm{R}^{2}=0.97$. This relation is named as the potential model estimation of SWC (SWCpot).

Finally, Gil Rodríguez et al. (2013) used an exponential function. The fitting of this function was based on the experimental data obtained in the laboratory using a soil sample obtained in the field plot of this study (Eq. (5)).

$S W C=k e^{-c T_{\text {cum }}}$

This relation is named as the exponential model estimation of SWC (SWCexp). The calculated numerical parameters were $\mathrm{k}=3.2$ and $\mathrm{c}=0.00165$, while $\mathrm{R}^{2}=0.98$.

The aforementioned numerical parameters were calculated for each SWC estimation model using the Generalized Least Squares method and the data of SWC and $\mathrm{T}_{\text {cum }}$ collected in the experimental site described in the previous section with the routine presented in Section 2.3.

\subsection{Experimental runs}

Temperature monitoring with DTS were conducted in the experimental site on August 2012 at 12:59, 14:23 and 16:49 h and no rainfall or irrigation events were present. The experimental procedure (see Fig. 4) was as follows: (1) before the heat pulse, temperature was recorded for $5 \mathrm{~min}$ and the average used as base temperature; (2) application of $19.40 \mathrm{~W} \mathrm{~m}^{-1}$ heat pulse during $122 \mathrm{~s}$ (heating phase), and (3) record the temperature for $15 \mathrm{~min}$ (cooling phase). The data were recorded every $2 \mathrm{~s}$.

The DTS unit was calibrated with the procedure proposed by the manufacturer since no extreme environmental conditions or damages were observed. This is the internal calibration that uses the data from a temperature probe (see Fig. 3), submerged in the calibration bath, to match the temperature measured in the section of the FO submerged in the calibration bath as well.

\subsection{Descriptive statistics}

For each SWC and $\mathrm{T}_{\text {cum }}$ data set, the first four statistical moments (average, variance, kurtosis and asymmetry —-skewness-) were calculated to study their similitude with a Gaussian distribution.

The same calculations were performed on each set of SWC and $\mathrm{T}_{\text {cum }}$ measurements after differentiating the series at several non-overlapping lags, from 1 till 128, working with a length of 1025 measures, approximately $133 \mathrm{~m}$. In this way, we could study the statistical moments of the frequency distribution of the values obtained in each lag. When a differentiation with lag 1 was applied, the statistics were calculated on 1024 values for each variable. In the case of differentiation with lag
128 , the number of values obtained was 8 .

For all these calculations, XLStat-Pro software (Addinsoft, New York) was used. This is a statistical package for Microsoft Excel available since 1993 .

\subsection{Multiscaling analysis}

The structure function analysis basically consists of studying the scaling behavior of the non-overlapping fluctuations of a variable for different scale increments. The statistical moments of these fluctuations are estimated, which depend only on the scale increment (Monin and Yaglom, 1975).

For non-stationary processes the Generalized Structure Function (GSF) of order $\mathrm{q}$ is defined as the $\mathrm{q}^{\text {th }}$ moment of the increments of initial values $\mu(i)$ The equation is:

$M_{q}(\Delta i) \equiv\left\langle|\mu(i+\Delta i)-\mu(i)|^{q}\right\rangle$

where $\mathrm{i}$ denotes the $\mathrm{i}^{\text {th }}$ data point, and $<>$ is the ensemble average. GSF are generalized correlation functions, which is particularly evident from Eq. (6) for the case of $q=2$. In general, q may be any real number not just integers, and can even be negative. However, there are divergence problems inherent to the negative-order exponent so that computations are best restricted to positive real number (Davis et al., 1994). If the process $\mu(i)$ is scale-invariant and self-similar or self-affine over some range of space lags $\left(\Delta \mathrm{i}_{\text {min }} \leq \Delta \mathrm{I} \leq \Delta \mathrm{i}_{\text {max }}\right)$, then the $\mathrm{q}^{\text {th }}$-order structure function is expected to scale as:

$M_{q}(\Delta i)=C_{q} \Delta i^{\zeta(q)} \approx \Delta i^{\zeta(q)}$

where $\mathrm{C}_{\mathrm{q}}$ can be a function of $\Delta \mathrm{i}$ which varies more slowly than any power of $\Delta \mathrm{i}$, and $\zeta(q)$ is the exponent of the structure function. $\zeta(q)$ is a monotonically non-decreasing function of q if $\mu(i)$ has absolute bounds (Frisch, 1995; Marshak et al., 1994). From Eq. (7) we can see that the statistics of the fluctuations over space lags $\Delta \mathrm{i}$ has two components; the first because it depends on the fluctuations at low $\Delta \mathrm{i}$ values, the second because of the scaling relation between the fluctuations and $\Delta \mathrm{i}$.

The behavior described by Eqs. (6) and (7) is called "multiscaling" because each statistical moment is scaling but with a different exponent. Therefore, a hierarchy of exponents can be defined using $\zeta(q)$ Eq. (8).

$H(q)=\frac{\zeta(q)}{q}$

where $\mathrm{H}(\mathrm{q})$ is the generalized Hurst exponent or self-similarity scaling exponent (Davis et al., 1994). Calculation of H(q) allows the straightforward identification of persistence, or long-space correlation, as well as the stationary/non stationary and monofractal/multifractal nature of the data (Lovejoy et al., 2001). 


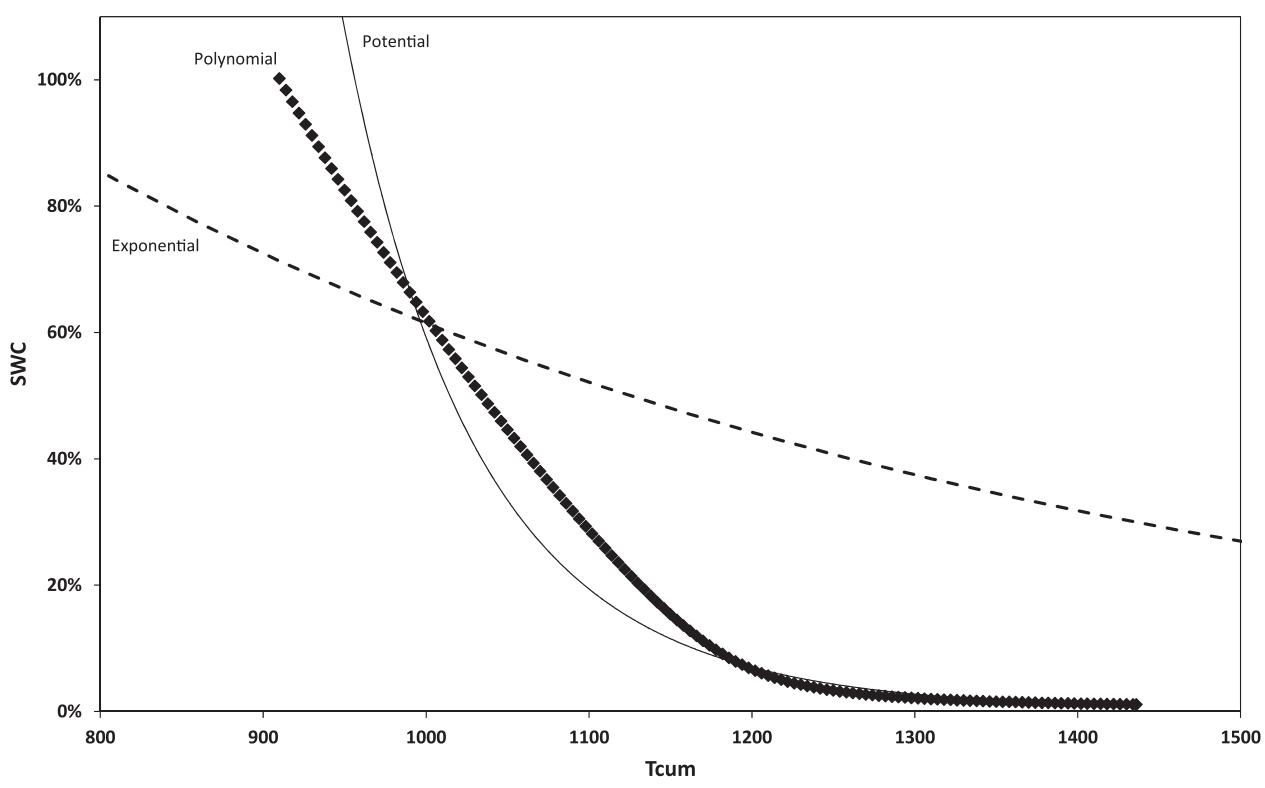

Fig. 5. Polynomial (dots), exponential (dashed line) and potential (continues line) functions adjusted to Tcum versus SWC.
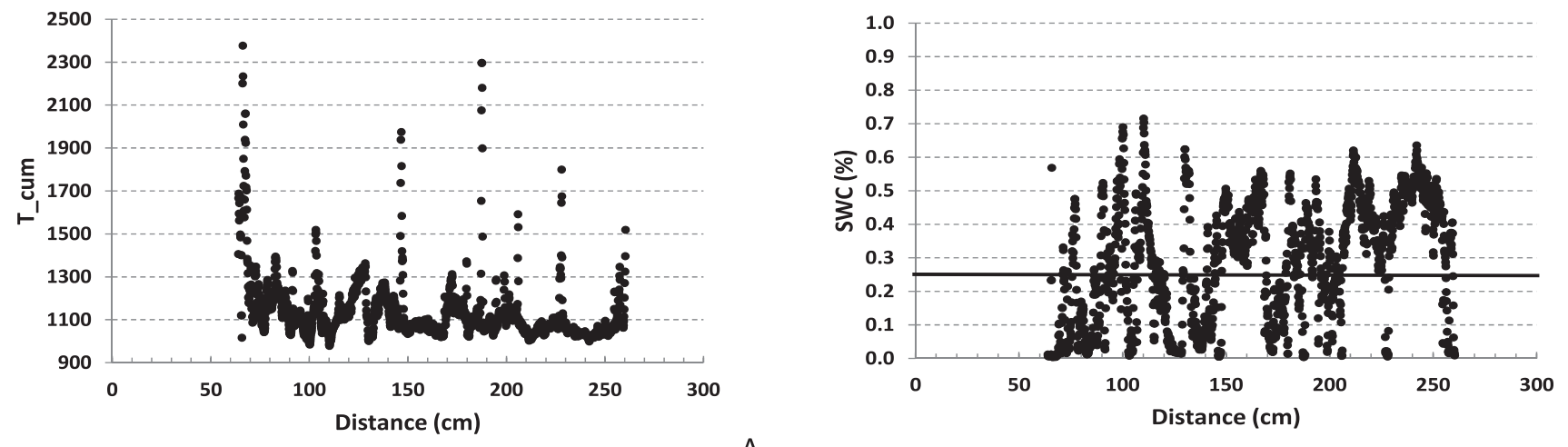

A
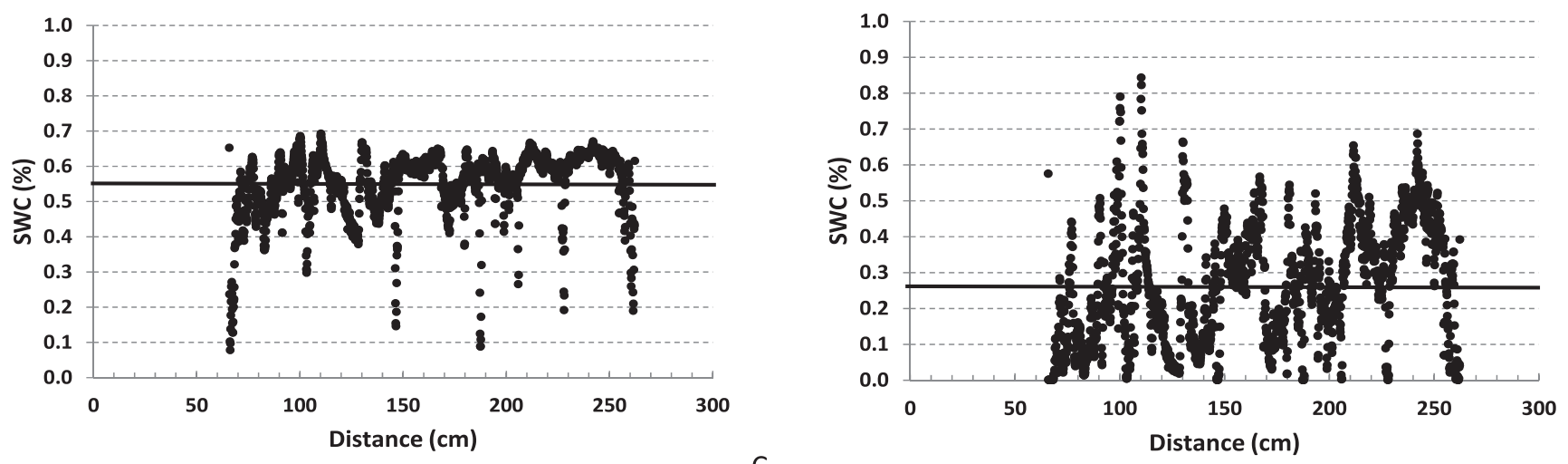

C

Fig. 6. Tcum data (A) and the soil water content (SWC) obtained applying three different adjustments: polynomial (B), exponential (C) and potential (D). Continuum line points the average value of the SWC series.

Stationary processes have scale-independent increments and $\zeta(q)=H(q) \equiv 0$, due to the invariance under translation. Processes with a linear $\zeta(q)$ (or a constant $\mathrm{H}(\mathrm{q})$ ) are non-stationary and monofractal, otherwise they are non-stationary and multifractal.

\section{Results and discussion}

\subsection{SWC estimation by fitting curves}

The fitting curves used in this study (polynomial - SWCpoly-, potential - SWCpot - and exponential - SWCexp) are shown in Fig. 5. All of them yielded a high determination coefficient $\left(R^{2} \geq 0.97\right)$, highlighting the goodness of the fit. However, the exponential and 

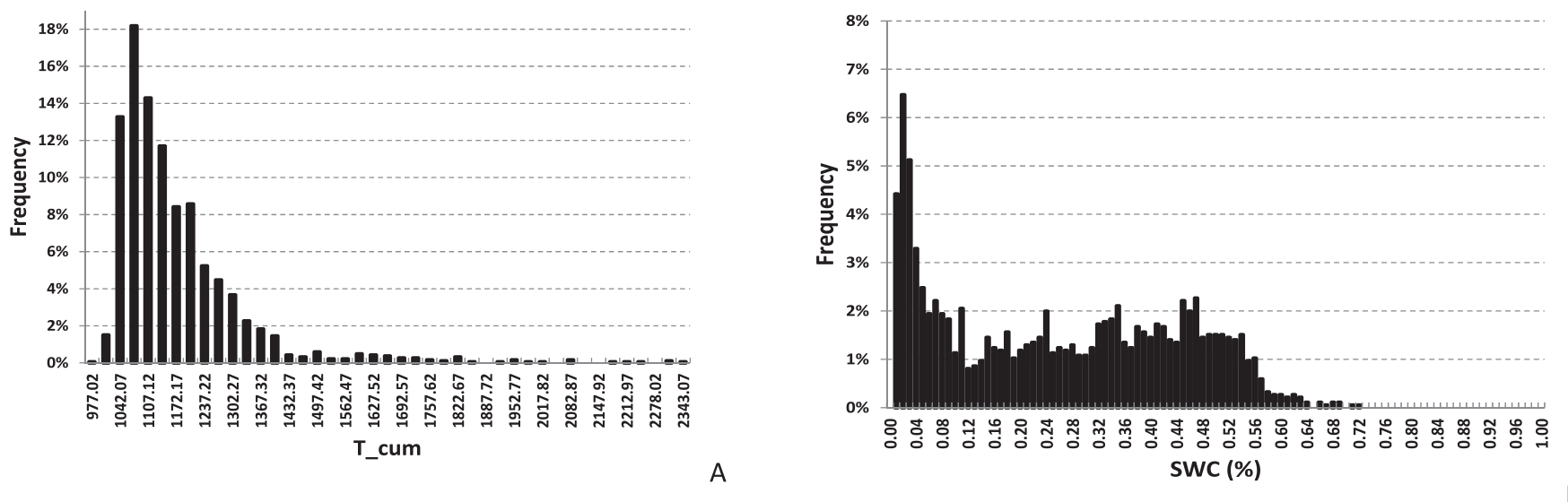

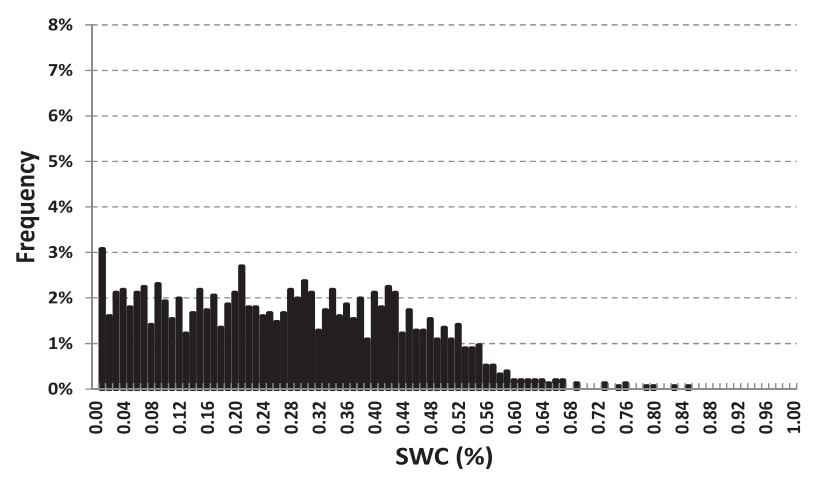

Fig. 7. Histograms: Tcum data (A) and the soil water content (SWC) obtained applying three different adjustments: polynomial (B), exponential (C) and potential (D).

Table 1

Descriptive statistics based on the first fourth moments (average, variance, kurtosis and asymmetry) on SWC series, obtained with three different adjustment, and T_cum data. Maximum and minimum values as well as the range are showed for each series.

\begin{tabular}{lllll}
\hline Statistics & \multicolumn{2}{l}{ SWC } & & \multirow{2}{*}{ Tcum } \\
\cline { 2 - 3 } & Polynomial & Exponential & Potential & \\
\hline Average & $25.30 \%$ & $55.10 \%$ & $26.70 \%$ & 1164.43 \\
Variance & 0.034 & 0.0086 & 0.0281 & 28070.169 \\
Coefficient of variation & 0.72 & 0.16 & 0.62 & 0.14 \\
Kurtosis & -1.28 & 4.94 & -0.66 & 15.92 \\
Assymetry & 0.17 & -1.89 & 0.28 & 3.43 \\
Range & $71.10 \%$ & $61.50 \%$ & $84.30 \%$ & 1398.578 \\
Minimum & $0.30 \%$ & $7.70 \%$ & $0.00 \%$ & 977.022 \\
Maximum & $71.50 \%$ & $69.20 \%$ & $84.30 \%$ & 2375.6 \\
\hline
\end{tabular}

potential curves corresponded to the data set from a soil column experiment (disturbed soil) over a wide range of SWC, and the other from data gathered in the field (no disturbed soil), within a narrower range of SWC.
As Fig. 5 shows, there are large differences among the three functions, more accentuated within the low range of SWC, which may lead to significant differences in the SWC estimation. The slope of the exponential function is lower for most SWC range except for values close to the residual moisture. Although this function showed a higher point density across a broader SWC range, the polynomial function covers it entirely. Either the potential or the polynomial estimations alike except for SWC beyond $60 \%$ where slight differences in $\mathrm{T}_{\text {cum }}$ yields to higher SWC estimations in the first.

Fig. 6A shows the original $\mathrm{T}_{\text {cum }}$ data obtained from the field installation, and Fig. 6B-D show the SWC data estimated with the three fitting curves using these data. As it was expected, major differences are observed among the curves in spite of their higher $\mathrm{R}^{2}$ coefficients. The shapes of SWCpoly and SWCpot were similar with an average value about $26 \%$, in contrast to SWCexp, whose average value was over $50 \%$, and it also showed a distinctive peak within the lower SWC range.

Thus, Fig. 7 shows the histograms for the four series $\left(\mathrm{T}_{\text {cum }}\right.$ and the SWC using the three functions). The histograms highlight that SWCexp reproduces better the $\mathrm{T}_{\text {cum }}$ behavior: both show a positive and large kurtosis values, equivalent skewness patterns, and similar relative dispersion. Conversely, SWCpoly and SWCpot present shapes quite

Table 2

Descriptive statistics using the first fourth moments (average, variance, asymmetry and kurtosis) of the differences in $\mathrm{T}_{-}$cum at different lags.

\begin{tabular}{|c|c|c|c|c|c|c|c|c|}
\hline Lag & 1 & 2 & 4 & 8 & 16 & 32 & 64 & 128 \\
\hline Distance $(\mathrm{cm})$ & 13 & 26 & 52 & 104 & 208 & 416 & 832 & 1664 \\
\hline Average & 0.328 & 0.655 & 1.311 & 2.622 & 5.243 & 10.487 & 20.973 & 36.567 \\
\hline Variance & 2790.52 & 8177.05 & 20289.25 & 22293.76 & 37461.90 & 23633.23 & 13816.42 & 26122.40 \\
\hline Coefficient of variation & 161.05 & 138.05 & 108.65 & 56.94 & 36.91 & 14.66 & 5.65 & 4.54 \\
\hline Kurtosis & 47.1 & 25.77 & 22.24 & 15.51 & 13.82 & 5.92 & 0.47 & -0.76 \\
\hline Assymetry & -2.76 & -1.09 & -1.34 & -0.39 & 0.58 & 1.51 & 0.1 & -0.21 \\
\hline Data points & 1024 & 512 & 256 & 128 & 64 & 32 & 16 & 8 \\
\hline
\end{tabular}


Table 3

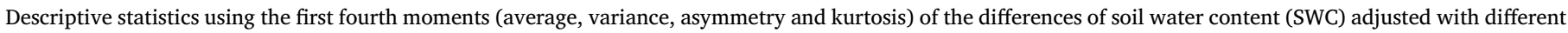
functions and at different lags.

\begin{tabular}{|c|c|c|c|c|c|c|c|c|}
\hline Lag & 1 & 2 & 4 & 8 & 16 & 32 & 64 & 128 \\
\hline Distance $(\mathrm{cm})$ & 13 & 26 & 52 & 104 & 208 & 416 & 832 & 1664 \\
\hline Data points & 1024 & 512 & 256 & 128 & 64 & 32 & 16 & 8 \\
\hline \multicolumn{9}{|l|}{ SWCpoly } \\
\hline Average & -0.0004 & -0.0007 & -0.0014 & -0.0029 & -0.0058 & -0.0115 & -0.0231 & -0.0108 \\
\hline Variance & 0.0021 & 0.0054 & 0.0129 & 0.0242 & 0.0429 & 0.0518 & 0.035 & 0.0642 \\
\hline Coefficient of variation & -114.56 & -104.98 & -81.12 & -53.64 & -35.71 & -19.79 & -8.09 & -23.46 \\
\hline Kurtosis & 31.2 & 6.4 & 2.7 & 1.8 & 0.8 & 0.9 & -0.3 & -0.4 \\
\hline Assymetry & 1.1 & -0.1 & 0 & -0.3 & -0.2 & 0.5 & 0.5 & 1 \\
\hline \multicolumn{9}{|l|}{ SWCexp } \\
\hline Average & 0.0001 & 0.0003 & 0.0006 & 0.0011 & 0.0023 & 0.0045 & 0.0091 & -0.0056 \\
\hline Variance & 0.0007 & 0.0023 & 0.0061 & 0.0107 & 0.0161 & 0.0177 & 0.0319 & 0.0414 \\
\hline Coefficient of variation & 264.57 & 159.86 & 130.17 & 94.03 & 55.16 & 29.56 & 19.62 & -36.33 \\
\hline Kurtosis & 70.7 & 40.9 & 17 & 6.8 & 5.8 & 4.9 & 2.1 & 1.5 \\
\hline Assymetry & 4 & 2.9 & 1.5 & 0.5 & 0.2 & -1.1 & -1 & -1.2 \\
\hline \multicolumn{9}{|l|}{ swCpot } \\
\hline & 0.0004 & 0.0008 & 0.0017 & 0.0033 & 0.0067 & 0.0134 & 0.0268 & 0.057 \\
\hline Variance & 0.0017 & 0.0052 & 0.0125 & 0.0255 & 0.0521 & 0.0685 & 0.0886 & 0.1384 \\
\hline Coefficient of variation & 103.07 & 90.13 & 65.76 & 48.39 & 34.06 & 19.53 & 11.1 & 6.52 \\
\hline Kurtosis & 41.3 & 12.1 & 5 & 2.3 & 1.5 & 0.5 & 0.2 & -1.4 \\
\hline Assymetry & 2.5 & 0.6 & 0 & 0 & -0.1 & 0 & -0.1 & 0 \\
\hline
\end{tabular}

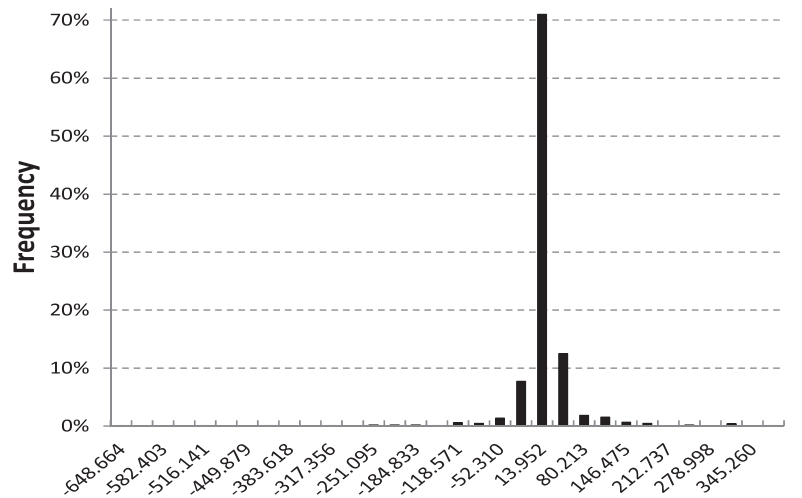

$\Delta$ T_cum

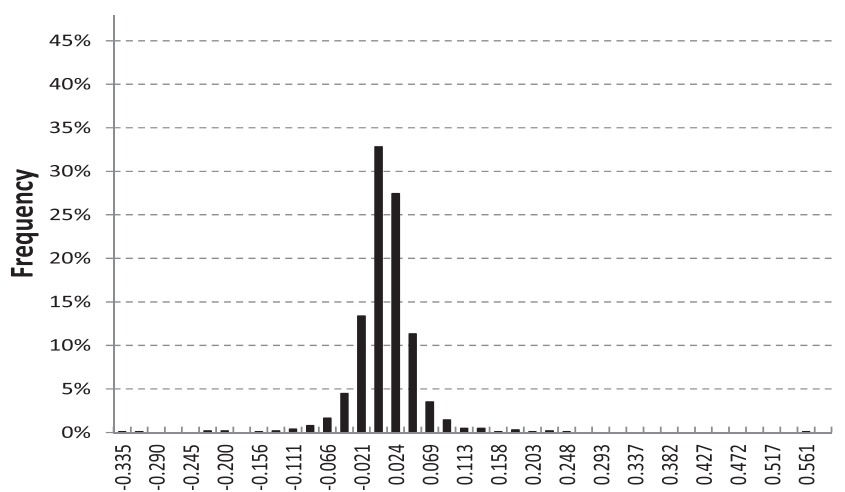

$\Delta$ SWC

A

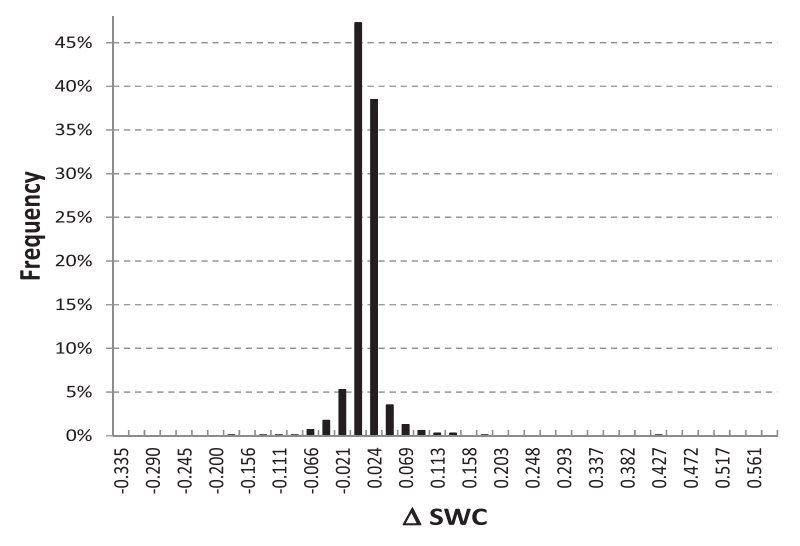

C

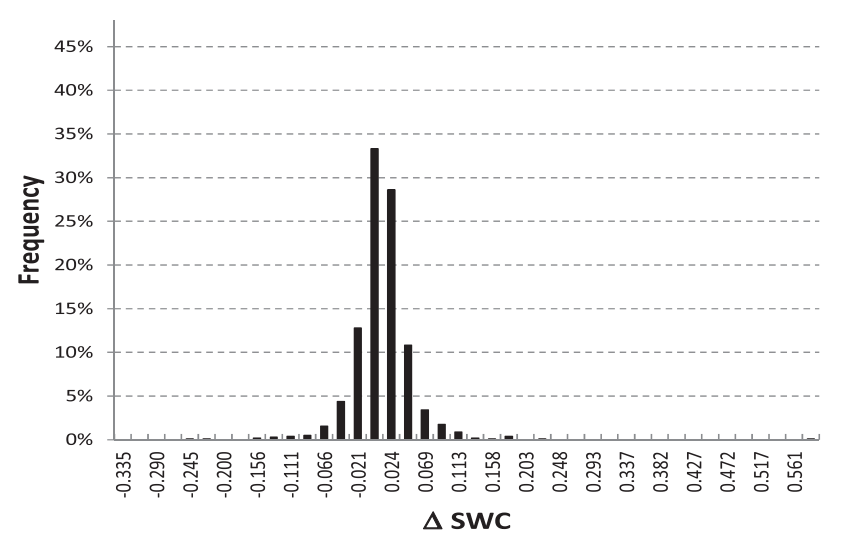

Fig. 8. Histograms (differentiated series at lag 1): Tcum data (A) and the soil water content (SWC) obtained applying three different adjustments: polynomial (B), exponential (C) and potential (D).

different of $\mathrm{T}_{\text {cum }}$.

Summarizing the histograms displayed in Fig. 7, the information given in Table 1 points to the existence of non-Gaussian behavior in the $\mathrm{T}_{\text {cum }}$ which presents a distribution with the values concentrated more around the mean value (kurtosis), and with a greater skewness than would usually correspond to a Gaussian distribution. None of the fitting curves presented such an extreme values of kurtosis and skewness so the non-Gaussian behavior of the $\mathrm{T}_{\text {cum }}$ seems to be blurred by the SWC estimation procedure. 
Table 4

Generalized Hurst exponents values () from the two extremes, $q=0.25$ and $q=4$, and for $q=1$ () for soil water content (SWC) adjusted with different functions and T_cum. is the amplitude of variation of. Estimations have been made through the generalized structure function (GSF).

\begin{tabular}{lllll}
\hline Data & $\mathrm{H}(0.25)$ & $\mathrm{H}(4)$ & $\Delta \mathrm{H}$ & $\mathrm{H}(1)$ \\
\hline T_cum & 0.585 & 0.345 & 0.2399 & 0.535 \\
SWC polynomial & 0.583 & 0.320 & 0.2630 & 0.541 \\
SWC exponential & 0.515 & 0.249 & 0.2659 & 0.499 \\
SWC potential & 0.564 & 0.316 & 0.2178 & 0.518 \\
\hline
\end{tabular}

\subsection{Statistics on fluctuations}

Focusing our attention on the differentiated values at different lags (Tables 2 and 3), we observed that lags larger than 64 lacked enough data to provide a good estimation of the statistical moments; therefore, we concentrated on the lags from 1 to 64 . From Table 2, we observe that for 1 and 2 lags $(0.13$ and $0.26 \mathrm{~m})$, the averages are almost zero and increase from lags 4-64 (from $0.52 \mathrm{~m}$ to $8.32 \mathrm{~m}$ ). These figures indicate that at that range of scales, the trends were revealed and were positive.

The non-Gaussian performance previously observed can also be perceived in the results for different lags (see Table 2). The statistics on the $T_{\text {cum }}$ series differences at lag 1 (Table 2) point out a high kurtosis, as it is perceived in Fig. 8A, and asymmetry significant different from a Gaussian distribution. Both statistical moments change their values as lag is increasing presenting Gaussian statistical moments at lag 64 . Kurtosis decreases from lag 1 to 6 , from 47.10 to 0.47 , and asymmetry increases from -2.76 to 0.10 .

Apparently, physical elements do not justify the non-Gaussian patterns in the plot. Care was taken to maintain the installation, in bare soil, for two years to ensure adequate assembly of the entire cable with the ground. Likewise, the field plot had a uniform soil and almost zero slope and also, no water was applied to the field during the experimental runs. However, the results on the thermal properties don't

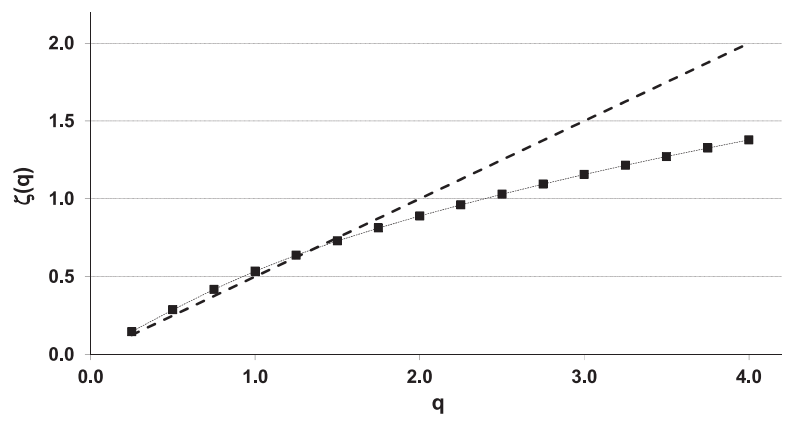

A

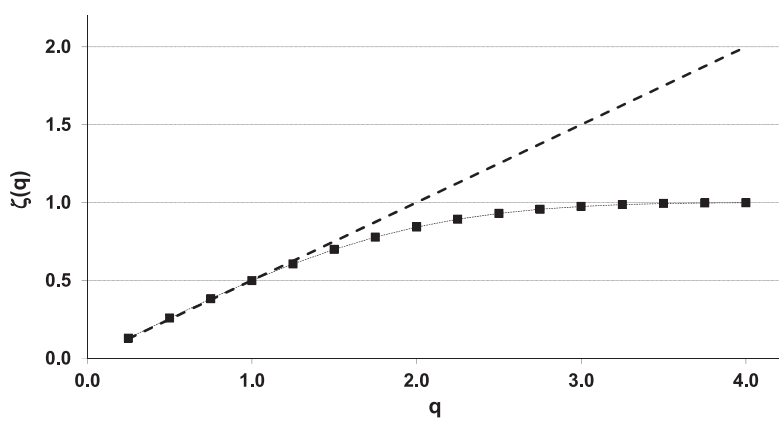

follow a Gaussian pattern that may highlight a no correlated error. We must recall here different works commenting the need of additional calibration, as van de Giesen et al (2012) or Haussner et al. (2011) detected, or the possible presence of hysteresis effects in the measurement, as observed by Sourbeer and Loheide (2016), among others.

Moreover, a distinctive behavior is found applying the same analysis to the SWC series, adjusted with the three functions (Table 3).

In this case the SWCexp function does not properly replicate the behavior observed in the $\mathrm{T}_{\text {cum }}$. It apparently contradicts the results presented in Table 1 and Fig. 7 concerning the absolute descriptive statistics. The kurtosis, the asymmetry and the relative dispersion are superior in SWCexp than in the $\mathrm{T}_{\text {cum }}$. Although the potential function seems to better reproduce the $\mathrm{T}_{\text {cum }}$ patterns at different scales, it can also be perceived that tends to hide the possible existence of nonGaussian patterns observed in the $\mathrm{T}_{\text {cum }}$.

SWCpoly average has a negative value at all lags and a decreasing trend, meanwhile SWCexp and SWCpot average presents positive values and increasing trend. As in the $\mathrm{T}_{\text {cum }}$ case, this indicates that at that range of scales the trends were positive for SWCexp and SWCpot, and negative for SWCpoly. As it happened with $\mathrm{T}_{\text {cum }}$ (see Table 2), the coefficient of variation decreases as the lag increments.

Respect to the kurtosis, for all the SWC differentiated series there is a common decrease in value with higher lags although they present slight differences. Although SWCpot present similar kurtosis at lag 1 that $\mathrm{T}_{\text {cum }}$, none of the SWC fitting curves seem to replicate properly the $\mathrm{T}_{\text {cum }}$ behavior for all the lags: opposite to SWCpot and SWCpoly, SWCexp show higher density of values around the average at every lag.

As listed in Table 4, the asymmetry values at lag 1 for the SWC differentiated series are higher than 1. From lag 2 to lag 64, SWCpoly and SWCpot asymmetry reduce to assumable Gaussian values meanwhile SWCexp keeps it higher than 1 till lag 8. None of the SWC fitting curves asymmetry replicates properly the $\mathrm{T}_{\text {cum }}$ behavior either: contrary to SWCpot and SWCpoly, present more extreme values than $\mathrm{T}_{\text {cum }}$.

Observing the combinations of kurtosis and asymmetry at each lag for SWCpoly and SWCpot series, there was always a lag (from lag 16 to lag 64) at which the obtained values are very close to those obtained
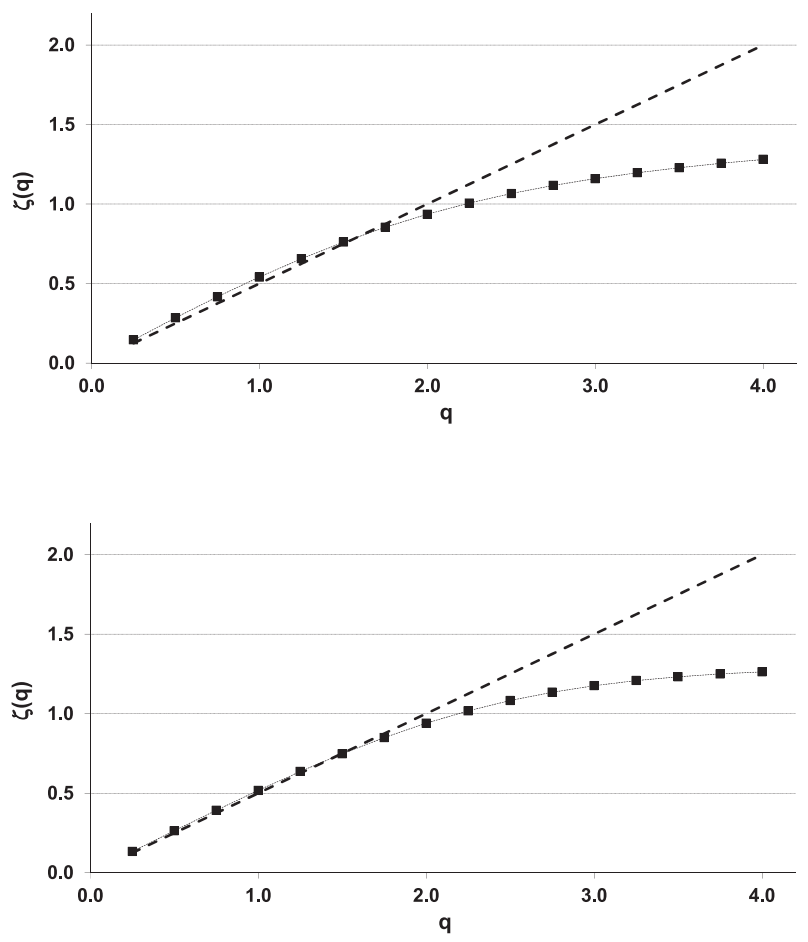

Fig. 9. curves obtained by GSF: A) T_cum, B) SWC adjusted with a polynomial function, C) SWC adjusted with an exponential function and D) SWC adjusted with an potential function. Continuous lines are straight lines with slope 0.5 representing a non-correlated noise. 

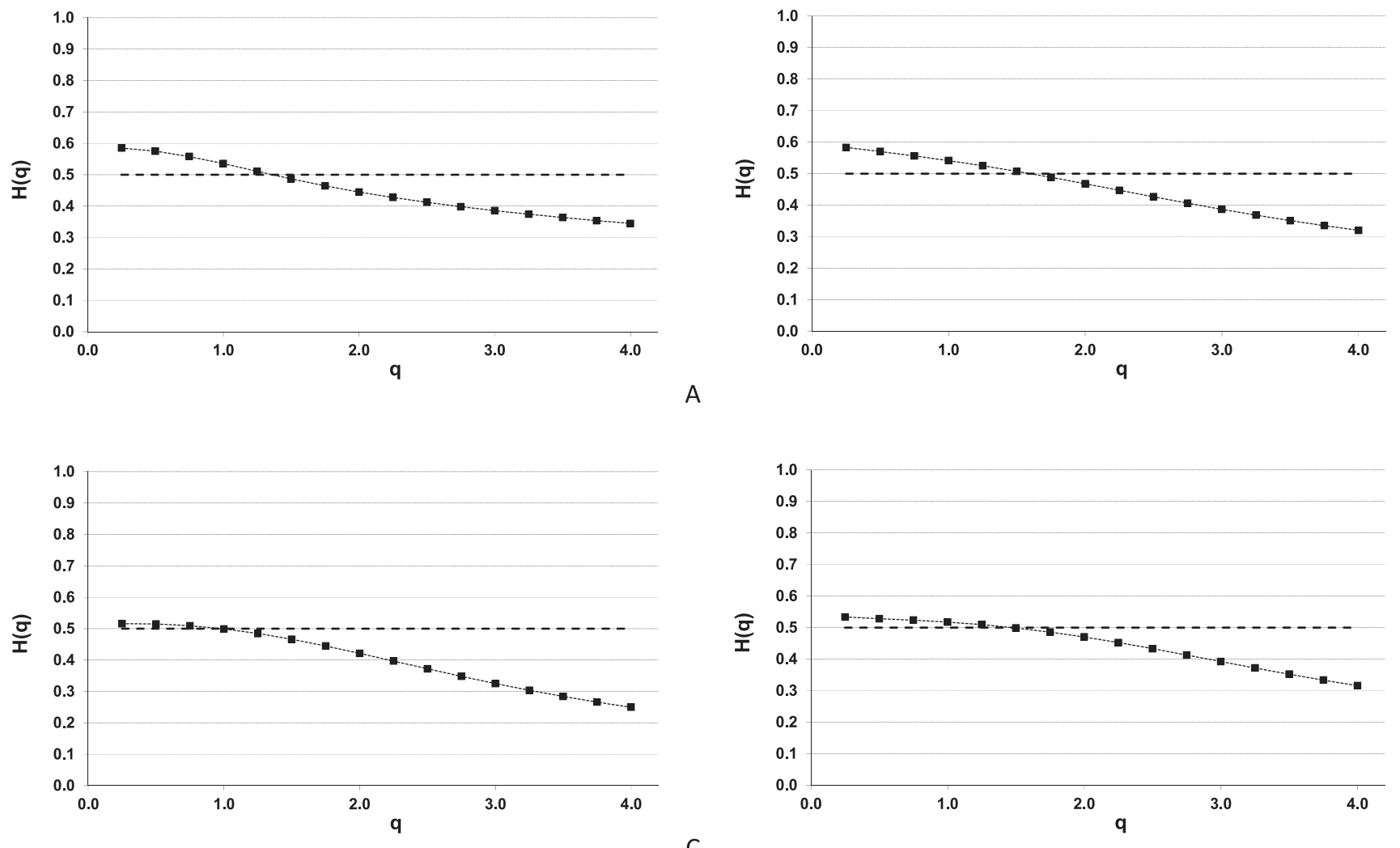

C

Fig. 10. Generalized Hurst exponent: A) T_cum, B) SWC adjusted with a polynomial function, C) SWC adjusted with an exponential function and D) SWC adjusted with an potential function. Continuous lines are straight lines with slope 0 corresponding to non-correlated noise with Hurst value 0.5 .

from a Gaussian distribution (Table 3). Therefore, if the SWC measurements were estimated at that resolution using these two approaches, the SWC increments would be represented by a Gaussian distribution; otherwise, simple fractional Brownian motion modelling would be chosen. Furthermore, SWCexp does not present statistics compatible with a Gaussian distribution at any lag.

Another important point is that each statistical moments (variance, kurtosis and asymmetry) do not present a linear relationships with lags or distance and show different magnitudes making a different scaling for each statistical moment. This implies a multifractal character.

By obtaining the measurements using this technology at higher resolutions, the probability distributions of transects' SWC increments were revealed to be quite symmetrical and to have heavy tails that described a non-Gaussian probability distribution, and a more complex scaling model is needed. Other authors have pointed this out in several contexts (Guadagnini et al., 2012, 2015).

\subsection{Multiscaling analysis}

The GSF obtained from the four series studied, with a total number of 1025 values, are showed in Fig. 9, where the maximum lag chosen was 64 data points ( $L=\Delta \mathrm{i}_{\max }$ ), an equivalent to $8.32 \mathrm{~m}$, and in all the linear regressions estimation a minimum $\mathrm{R}^{2}$ of 0.90 for all the $\mathrm{q}$ used.

The results obtained from the $\zeta(q)$ curves corroborate the multifractal nature of the series studied in this work. The intensity of this multiscaling differs among SWC curves and between them and $\mathrm{T}_{\text {cum }}$ as the different slope of the curves presented in Fig. 9 shows. The asymptotic shape of these curves point out that it is better to quantify the Hurst parameter, as its amplitude, to further study this first observation.

For comparison, we also plotted in Fig. 9 the line $\zeta(q)=\frac{q}{2}$, which corresponds to Brownian motion or a noise without any correlation (Morató et al., 2017). In the four cases, the $\zeta(q)$ function is significantly different from the Brownian line. It highlights a correlation of the increments among several scales. Although each SWC function is close to the straight line at low $\mathrm{q}$ values, the curves move away from the Gaussian line as $\mathrm{q}$ increases. Graphics also show that, compared to $\mathrm{T}_{\text {cum }}$, SWCpoly and SWCpot tend to overestimate the presence of the Gaussian pattern as q increases, contrary to SWCexp. The graphics included in Fig. 9 also point that SWCexp does not represent properly the multifractal nature of $\mathrm{T}_{\text {cum }}$.

The generalized Hurst exponents derived from the $\zeta(q)$ function based on GSF are showed in Fig. 10, where the straight line represents the case of pure noise or uncorrelated noise $(\mathrm{H}(\mathrm{q})=0.5)$. At lower $\mathrm{q}$ values, the $\mathrm{H}(\mathrm{q})$ is closer to 0.5 and decreases obtaining an antipersistent character in the higher moments. To quantify the variation of $\mathrm{H}(\mathrm{q})$ versus $\mathrm{q}$, we have subtracted the extreme values $(\mathrm{H}(0.25)-\mathrm{H}(4))$ for each variable (see Table 4). The highest amplitude of $\mathrm{H}(\mathrm{q})$ corresponds to SWCpoly, followed by SWCpot and SWCexp.

All the series studied, $\mathrm{T}_{\text {cum }}$ and the three SWC obtained with different adjustment, present a degree of non-conservation lower than one $(H=\zeta(q=1)=H(q=1))$ and therefore it is not necessary to apply a detrending fluctuation analysis (Kantelhardt et al., 2002).

At lower q values, the $\mathrm{T}_{\text {cum }}$ shows a persistent behavior related to variations of soil properties at high spatial scales and with positive correlations. Conversely, the series behave antipersistent for large $\mathrm{q}$ values. Although there does not seem to be differences according to the values presented in Table 4, the graphics included in Fig. 10 show relevant differences between $\mathrm{T}_{\text {cum }}$ and each SWC estimation. Otherwise, SWCexp and SWCpot do not faithfully represent the character of the correlations observed in the $\mathrm{T}_{\text {cum }}$. Both these assessments and those formulated in view of Fig. 8 point to the relevant information loss in the exponential fitting curve procedure as a result of having used disturbed samples to obtain the fitting curve. Complementarily, SWCpoly seem to better replicate the $\mathrm{T}_{\text {cum }}$ results.

With regards to the multiscaling analysis, the behavior of the $\mathrm{T}_{\text {cum }}$ 
again points to the possible existence of interference from the equipment or the measurement procedure. The presence of persistent patterns for small q values could mark that the calibration procedure need additional steps to better balance the slope of the temperature measurement along the FO cable. In any case, additional studies should be carried out in order to contrast the possible influence of errors in the equipment or in the facilities or of an inadequate calibration process.

\section{Conclusions}

The AHFO technique with DTS has made feasible to estimate the soil water content SWC in the field, with a soil sampling of $0.125 \mathrm{~cm}$, from a transect $133 \mathrm{~m}$ long, through the so called cumulated temperature variable $\mathrm{T}_{\text {cum }}$. The relation $\mathrm{T}_{\text {cum }}$ versus SWC was estimated by three fitting functions: potential, exponential and polynomial.

The three $\mathrm{T}_{\text {cum }}$-SWC functions showed a high determination coefficient $\mathrm{R}^{2}>0.97$ although their SWC estimations for the same soil differ. The SWCexp fitting curve better represent the Tcum properties in view of the absolute statistical moments. Conversely, although these metrics suggest the presence of non-Gaussian patterns in the Tcum, none of the fitting curves properly replicate such behavior.

The scaling analysis of the statistics at different lags also denotes the presence of patterns that move away from the Brownian behavior at small lags, while at the highest lags the results are comparable with a Gaussian behavior. This fact is important when a model should simulate SWC at small distances as the heterogeneity in the field has a huge influence in it. The results from the analysis of the structure function show the multifractal character of $\mathrm{T}_{\text {cum }}$ data.

In general terms, the evolution of the Tcum, suggest that certain elements not properly managed during the DTS measurement could cause the non-Gaussian behavior. Additional research must be conducted in order to identify the source of these interferences. Regarding the SWC estimation, the results allow concluding that none of the analyzed SWC fitting curves replicates very precisely the Tcum behavior. It may also be considered proven the relevant influence of the soil sample used for the fitting curve estimation since the curves deduced from undisturbed soils better reproduce the Tcum behavior.

\section{Acknowledgements}

We would like to thank the Commission of Science and Technology (CICYT) for the funding received for this research through project AGL2004-01689.

\section{References}

Benitez-Buelga, J., Rodríguez-Sinobas, L., García-Calvo, R., Gil-Rodríguez, M., Sayde, C., Selker, J., 2016. Calibration of soil moisture sensing with subsurface heated fiber optics using numerical simulation. Water Resour. Res. 52.

Burrough, P.A., Bouma, J., Yates, S.R., 1994. The state of the arts in pedometric Geoderma 62, 311-326.

Dabach, S., Shani, U., Lazarovitch, M., 2015. Optimal tensiometer placement for highfrequency subsurface drip irrigation management in heterogeneous soils. Agric. Water Manage. 152, 91-98.

Davis, A., Marshak, A., Wiscombe, W., Cahalan, R., 1994. Multifractal characterizations of nonstationarity and intermittency in geophysical fields: observed, retrieved, or simulated. J. Geophys. Res. 99, 8055-8072.

Dingman, S.L., 2002. Physical Hydrology. Prentice Hall, Upper Saddle River, N.J.

Fischer, G., Tubiello, F.N., van Velthuizen, H., Wiberg, D.A., 2007. Climate change impacts on irrigation water requirements: effects of mitigation, 1990-2080. Technol.
Forecast. Soc. 74 (7), 1083-1107.

Frisch, U., 1995. Turbulence. The Legacy of A. Kolmogorov. Cambridge University Press, Cambridge, UK.

Garcia Moreno, R., Diaz Alvarez, M.C., Saa Requejo, A., Valencia Delfa, J.L., Tarquis, A.M., 2010. Multiscaling analysis of soil roughness variability. Geoderma 160 (1), 22-30.

Gil Rodríguez, M., Rodríguez Sinobas, L., Benitez Buelga, J., Sánchez-Calvo, R., 2013. Application of active heat pulse method with fiber optic temperature sensing for estimation of wetting bulbs and water distribution in drip emitters. Agric. Water Manage. 120, 72-78.

Guadagnini, A., Riva, M., Neuman, S.P., 2012. Extended power-law scaling of heavytailed random air-permeability fields in fractured and sedimentary rocks. Hydrol. Earth Syst. Sci. 16, 3249-3260.

Guadagnini, A., Neuman, S.P., Nan, T., Riva, M., Winter, C.L., 2015. Scalable statistics of correlated random variables and extremes applied to deep borehole porosities. Hydrol. Earth Syst. Sci. 19, 729-745.

Haussner, M.B., Suarez, F., Glander, K.E., van de Giesen, N., Selker, J., Tyler, S.W., 2011. Calibrating single-ended fiber-optic raman spectra distributed temperature sensing. Sensors 11, 10859-10879.

Hurst, H.E., 1951. Long-term storage capacity of reservoirs. Trans. Am. Soc. Civ. Eng. 116, 770-799.

Kantelhardt, J.W., Zschiegner, S.A., Bunde, A., Havlin, S., Koscielny-Bunde, E., Stanley, H.E., 2002. Multifractal detrended fluctuation analysis of nonstationary time series. Phys. A 316, 87-114.

Kravchenko, A.N., Omonode, R., Bollero, G.A., Bullock, D.G., 2002. Quantitative mapping of soil drainage classes using topographical data and soil electrical conductivity. Soil Sci. Soc. Am. J. 66, 235-243.

Lopez de Herrera, J., Herrero Tejedor, T., Saa-Requejo, A., Tarquis, A.M., 2016. Effects of tillage on variability in soil penetration resistance in an olive orchard. Soil Res. 54 (2), 134-143.

Lovejoy, S., Schertzer, D., Stanway, J.D., 2001. Fractal behaviour of ozone, wind and temperature in the lower stratosphere. Phys. Rev. Lett. 86, 5200-5203.

Mandelbrot, B.B., 1982. The Fractal Geometry of Nature. W.H. Freeman, New York.

Marshak, A., Davis, A., Cahalan, R.F., Wiscombe, W.J., 1994. Bounded cascade models as non-stationary multifractals. Phys. Rev. E 49, 55-69.

Molinari, A., Guadagnini, L., Marcaccio, M., Guadagnini, A., 2015. Arsenic fractioning in natural solid matrices sampled in a deep groundwater body. Geoderma 247-248, 88-96.

Monin, A.S., Yaglom, A.M., 1975. Stratistical Fluid Mechanics: Mechanics of Turbulence. MIT Press, Boston.

Morató, M.C., Castellanos, M.T., Bird, N.R., Tarquis, A.M., 2017. Multifractal analysis in soil properties: spatial signal versus mass distribution. Geoderma 287, 54-65.

Nielsen, D., Wendroth, O., 2003. Spatial and Temporal Statistics-sampling Field Soils and Their Vegetation. GeoEcology textbook, Catena-Verlag, Reiskirchen.

Pozdnyakova, L., Giménez, D., Oudemans, P.V., 2005. Spatial analysis of cranberry yield at three scales. Agron. J. 97 (1), 49-57.

Sayde, C., Gregory, C., Rodríguez, M., Tufillaro, N., Tyler, S., Van de Giesen, N., English, M., Cuenca, R., Selker, J.S., 2010. Feasibility of soil moisture monitoring with heated fiber optics. Water Resour. Res. 46.

Schmitt, F., Lovejoy, S., Schertzer, D., 1995. Multifractal analysis of the Greenland icecore project climate data. Geophys. Res. Lett. 22 (13), 1689-1692.

Selker, J.S., Thévenaz, L., Huwald, H., Mallet, A., Luxemburg, W., van de Giese, N., Stejskal, M., Zeman, J., Westhoff, M., Parlange, B., 2006. Distributed fiber-optic temperature sensing for hydrologic systems. Water Resour. Res. 42.

Serna, J.L., Muñoz, J.F., Suárez, F., 2017. Assessment of the active method to determine soil moisture. Ingeniería del agua 21 (3), 165-178.

Sourbeer, J.J., Loheide, S.P., 2016. Obstacles to long-term soil moisture monitoring with heated distributed temperature sensing. Hydrol. Process. 30, 1017-1035.

Striegel, A.M., Loheide, P., 2012. Heated distributed temperature sensing for field scale soil moisture monitoring. Ground Water 50 (3), 340-347.

Taqqu, M.S., Teverovsky, V., Willinger, W., 1995. Estimators for long-range dependence: an empirical study. Fractals 3 (4) 785-198.

Tarquis, A.M., Bird, N.R., Whitmore, A.P., Cartagena, M.C., Pachepsky, Y., 2008. Multiscale analysis of soil transect data. Vadose Zone J. 7, 563-569.

van de Giesen, N., Steele-Dune, S.C., Jansen, J., Hoes, O., Haussner, M.B., Tyler, S., Selker, J.S., 2012. Double-ended calibration of fiber-optic raman spectra distributed temperature sensing data. Sensors 12, 5471-5485.

Vereecken, H., Kasteel, R., Vanderborght, J., Harter, T., 2007. Upscaling hydraulic properties and soil water flow processes in heterogeneous soils: a review. Vadose Zone J. 6, 1-28.

Webster, R., Oliver, M.A., 2001. Geostatistics for Environmenntal Scientists. Statistics in Practice. Willy, Chichester.

Zeleke, T.B., Si, B.C., 2004. Scaling properties of topographic indices and crop yield: multifractal and joint multifractal approaches. Agron. J. 96, 1082-1090. 